\title{
Association between fried food consumption and hypertension in Korean adults
}

\author{
Yunjin Kang and Jihye Kim* \\ Department of Medical Nutrition, Graduate School of East-West Medical Science, Kyung Hee University, \\ Yongin 446-701, Korea \\ (Submitted 15 March 2015 - Final revision received 18 August 2015 - Accepted 9 September 2015 - First published online 9 October 2015)
}

\section{Abstract}

The present study explored the relationships between fried food consumption and metabolic risk factors and hypertension in Korean adults. The study was based on the fifth Korean National Health and Nutrition Examination Survey between 2010 and 2011 . A total of 9221 Korean adults aged $\geq 19$ years were studied. Fried food consumption was assessed using a validated FFQ. Metabolic risk factors such as waist circumference, fasting plasma glucose (FPG), TAG, HDL-cholesterol and systolic and diastolic blood pressure (SBP and DBP) were measured. Hypertension was defined as SBP $\geq 140 \mathrm{mmHg}$, DBP $\geq 90 \mathrm{mmHg}$ or current use of antihypertensive medication. Adjusted OR for elevated blood pressure significantly increased in men (OR 1.62; $95 \%$ CI 1.11, 2.37; $P_{\text {trend }}=0.0447$ ) and women (OR 2.20; $95 \%$ CI 1.21 , 4.00; $P_{\text {trend }}=0.0403$ ) with a greater than twice a week consumption of fried food compared with those who rarely consumed fried food. However, fried food consumption was not associated with other metabolic risk factors (abdominal obesity, high FPG, hypertriacylglycerolaemia, low HDL-cholesterol and the metabolic syndrome). The adjusted OR for hypertension increased by 2.4-fold in women (OR 2.37; $95 \%$ CI 1·19, 4.72; $P_{\text {trend }}=0.0272$ ) with a greater than twice a week fried food consumption compared with those who rarely consumed it. No significant association was found between fried food consumption and hypertension in men. This study suggests that frequent fried food consumption is associated with hypertension in Korean women. Further studies are needed to investigate the effect of different types of fried foods on hypertension

\section{Key words: Fried food consumption: Hypertension: Blood pressure: Korean adults}

Hypertension is one of the major risk factors for CVD. It is an important global public health issue in Korea and worldwide ${ }^{(1)}$. According to data from the Korean National Health and Nutrition Examination Survey (KNHANES), hypertension is steadily increasing in Korean adults. The prevalence of hypertension increased from $28.9 \%$ for men and $25.5 \%$ for women between 2007 and 2009 to $32.4 \%$ for men and $28.4 \%$ for women between 2010 and $2012^{(2)}$. Previous studies have reported associations between some nutrients, food and food groups and blood pressure $(\mathrm{BP})^{(3-5)}$. In particular, a high-fat diet including trans-fats and SFA was associated with increased $\mathrm{BP}^{(6)}$.

Frying is a common and popular cooking method in Western countries. Frying involves changes in flavour, taste and colour ${ }^{(7)}$. Because of the absorption of fat, frying imparts crispiness and flavour and improves the palatability of foods ${ }^{(8)}$. However, fried food consumption has been reported to be associated with metabolic risk factors ${ }^{(9-11)}$ and the metabolic syndrome (MetS) ${ }^{(12)}$. Frequent fried food consumption is associated with larger waist circumference $(\mathrm{WC})^{(9)}$, elevated $\mathrm{BP}^{(10)}$, higher LDL-cholesterol level $^{(11)}$ and lower HDL-cholesterol level ${ }^{(9)}$. Although fried food intake has been found to be positively associated with metabolic risk factors including $\mathrm{BP}$, information on the relationship between fried food consumption and hypertension is very limited. To the best of our knowledge, only one study has reported that fried food consumption was positively associated with incident hypertension in a Spanish population ${ }^{(13)}$. However, the relationship between fried food consumption and hypertension has not been investigated in Asian countries, although frying food is also a common practice in Asia.

The Korean diet has recently undergone rapid changes. In particular, the consumption of Western-style food including fried food is rapidly increasing among Koreans ${ }^{(14)}$. However, the associations between fried food intake and metabolic risk factors and hypertension have not been investigated in a Korean population. Therefore, the aim of this study was to investigate the associations between fried food consumption, metabolic risk factors and hypertension in Korean adults, using nationally representative survey data.

Abbreviations: BP, blood pressure; DBP, diastolic blood pressure; DDS, dietary diversity score; FPG, fasting plasma glucose; MetS, metabolic syndrome; SBP, systolic blood pressure; TFA, trans-fatty acids; WC, waist circumference.

* Corresponding author: J. Kim, fax +82 31204 8119, email kjhye@khu.ac.kr 


\section{Methods}

\section{Study population}

The KNHANES is a national cross-sectional survey performed by the Korea Centers for Disease Control and Prevention (CDC) using a multistage, clustered, stratified and rolling sampling method. The survey contains a health interview, health examination and nutrition survey. We analysed the data in the KNHANES from 2010 to 2011, including 17476 (8958 in 2010 and 8518 in 2011) participants. A total of 13306 adults aged 19 years and older were selected for the present study. We excluded 4085 adults who reported unusual energy intakes $(<3347.2$ or $>16736 \mathrm{~kJ} / \mathrm{d}$ ( $<800$ or $>4000 \mathrm{kcal} / \mathrm{d}$ ) for men and $<2092$ or $>14644 \mathrm{~kJ} / \mathrm{d}$ ( $<500$ or $>3500 \mathrm{kcal} / \mathrm{d}$ ) for women) or with insufficient data in the socio-economic, anthropometric or biochemical information. As a result, 9221 adults (3634 men and 5587 women) were eligible for the analysis. The study was approved by the Korea CDC Institutional Review Board.

\section{Dietary intake assessment}

Fried food consumption was measured using a validated $F_{F Q}{ }^{(15)}$. The 109-item FFQ was administered twice, approximately 9 months apart. Four seasonal 3-d dietary records were used as a reference method. The average correlation coefficients measuring reproducibility were 0.54 for nutrients and 0.57 for food groups. The mean correlation coefficient measuring validity was 0.40 for all nutrients between the first FFQ and the dietary record. The FFQ was conducted through face-to-face interviews by trained dietitians. Participants were asked about usual frequencies of fried food consumption over the previous year as follows: 'How often did you eat fried food during the past year?'. We obtained data on usual food consumption during the past year on a daily, weekly or monthly basis. Answer options were presented in ten categories and classified into four groups: (1) rarely: none to 6-11 times/year; (2) $\geq 1 /$ month: 1 or 2-3 time(s)/month; (3) 1/week: 1 time/week; and (4) $\geq 2 /$ week: 2-6 times/week or 1-3 time(s)/d. The FFQ included all fried food regardless of food group. According to 24-h recall, fried meat including chicken, pork and beef accounted for $45 \%$ of all fried food and fried vegetable including onion, potato and pepper accounted for $31 \%$ of all fried foods. Fish and shellfish accounted for $12 \%$ of all fried food. The dietary intakes used to calculate nutrient intakes were determined using the 24-h recall method. Nutrient intakes were estimated from the food composition table of the Rural Development Administration in combination with the nutrient database of the Korea Health and Industry of Development Institute ${ }^{(16)}$.

\section{Measurements}

Height and body weight were measured as a part of the health examination study ${ }^{(17)}$. BMI was calculated as weight $(\mathrm{kg})$ divided by height squared $\left(\mathrm{m}^{2}\right)$. WC was measured by trained researchers as a part of the health examination study. WC was measured to the nearest $0.1 \mathrm{~cm}$ at the narrowest point between the lowest rib and the uppermost lateral border of the right iliac crest. BP was measured with a Baumanometer mercury sphygmomanometer
(WA Baum) after subjects had rested for $5 \mathrm{~min}$ in a sitting position. Systolic and diastolic blood pressures (SBP and DBP) were measured at phase I and V Korotkoff sounds ${ }^{(18)}$, respectively. Three readings of SBP and DBP were recorded, and the average of the last two readings was used for analysis. Blood samples were measured in the morning after an overnight fast. Fasting plasma glucose (FPG), TAG and HDL-cholesterol were measured enzymatically using a Hitachi automatic analyzer 7600 (Hitachi) in central, certified laboratories. All biochemical analyses were carried out within $2 \mathrm{~h}$ of blood sampling.

\section{Definition of the metabolic syndrome and hypertension}

The definition of the MetS came from the joint interim statement issued by the International Diabetes Federation and the American Heart Association/National Heart, Lung, and Blood Institute as $\geq 3$ of any of the following: abdominal obesity (WC $>90 \mathrm{~cm}$ for men or $>80 \mathrm{~cm}$ for women, as previously described); hyperglycaemia (FPG $\geq 100 \mathrm{mg} / \mathrm{dl}(5.5 \mathrm{mmol} / \mathrm{l})$ ) or current use of insulin or oral hypoglycaemia medication or a physician's diagnosis; hypertriacylglycerolaemia $\geq 150 \mathrm{mg} / \mathrm{dl} \quad(1.7 \mathrm{mmol} / \mathrm{l})$; low HDL-cholesterol $<40 \mathrm{mg} / \mathrm{dl} \quad(1.04 \mathrm{mmol} / \mathrm{l})$ in men or $<50 \mathrm{mg} / \mathrm{dl}(1.30 \mathrm{mmol} / \mathrm{l})$ in women; and elevated BP (SBP/DBP $\geq 130 / 85 \mathrm{mmHg}$ ) or the use of antihypertensive medication ${ }^{(19)}$. Hypertension was defined as SBP $\geq 140 \mathrm{mmHg}, \mathrm{DBP} \geq 90 \mathrm{mmHg}$ or current use of antihypertensive medication ${ }^{(20)}$.

\section{Covariates}

Demographic characteristics, socio-economic status and lifestyle factors were obtained using in-person interviews by a trained researcher and a self-administered questionnaire. Income level was categorised into three groups: low (below average); medium (average); and high (above average). Education level was divided into three groups: $\leq 6$ years (elementary school level); 6-12 years (middle or high school level); and $\geq 12$ years (college level). Smoking status was classified as non-smoker, former smoker or current smoker. Alcohol intake was categorised into non-drinker, moderate drinker ( $<2$ times) week) and heavy drinker $(\geq 2 \text { times/week })^{(21)}$. The regular physical activity group was classified into hard exercise for $\geq 20 \mathrm{~min}$ at a time $\geq 5 \mathrm{~d} /$ week, moderate exercise for $\geq 30 \mathrm{~min}$ at a time $\geq 5 \mathrm{~d}$ /week or walking for $\geq 30 \mathrm{~min}$ at a time $\geq 5 \mathrm{~d}$ /week. Chronic diseases such as CVD, type 2 diabetes and cancer were assessed by the question - Have you been diagnosed with a disease in the last year? (yes/no). Diet quality was assessed using a dietary diversity score (DDS). DDS was determined on the basis of foods the subjects consumed from the various five basic food groups (cereal, meat, dairy products, vegetable and fruit). When a subject consumed the designated amount of foods from a certain food group, one point was given. A maximum of five points was given when one ate the designated amount of foods from all five basic food groups ${ }^{(22)}$.

\section{Statistical analyses}

All data were analysed by PROC SURVEY using SAS version 9.3 (SAS Institute). To reflect national population estimates, sample 
weights were applied in all analyses. The Rao-Scott's $\chi^{2}$ test was used for categorical variables with the PROC SURVEYFREQ procedure. The PROC SURVEYMEANS procedure was used for means with their standard errors of continuous variables. The PROC SURVEYREG procedure was used to compare differences according to sex and frequency of fried food consumption. Interaction between sex and the frequency of fried food consumption was tested using likelihood ratio tests between the fully adjusted models and the same model, but by introducing the interaction product term.

A multivariable-adjusted logistic regression analysis was conducted to determine OR and $95 \%$ CI for components of the MetS and hypertension across levels of frequency of fried food consumption using PROC SURVEYLOGISTIC. For all the analyses, an unadjusted univariate model, and an age- and sex-adjusted model (model 1) and a multivariable model were used. Model 2 was adjusted for age, sex, BMI, income level, educational level, smoking status, physical activity, alcohol intake, energy intake, Na intake, DDS and disease (cancer, CVD or diabetes). For the selection of potential confounders in the multivariable model, and as currently recommended ${ }^{(23)}$, the previously published scientific literature, including our own results based on the cross-sectional study regarding potential risk factors for the MetS or hypertension, was taken into account, avoiding exclusively the statistical approach with the $P$ value, the step-wise procedures or the changes in point estimates after adjusting for potential confounders. All $P$ values $<0.05$ were considered to be statistically significant.

\section{Results}

\section{Characteristics of subjects}

Characteristics of study subjects are shown in Table 1. Women were older compared with men $(P=0 \cdot 0140)$. Men were more likely to be educated, current smokers, heavy alcohol drinkers and regular exercisers and to have higher energy intake and $\mathrm{Na}$ intake and a lower DDS compared with women. Men had

Table 1. Characteristics of the study subjects

(Numbers and percentages; mean values with their standard errors)

\begin{tabular}{|c|c|c|c|c|c|}
\hline & \multicolumn{2}{|c|}{ Men $(n$ 3634) } & \multicolumn{2}{|c|}{ Women ( $n$ 5587) } & \multirow[b]{2}{*}{$P^{*}$} \\
\hline & $n$ & $\%$ & $n$ & $\%$ & \\
\hline Age (years) & $44 \cdot 3$ & 0.4 & $45 \cdot 3$ & 0.4 & 0.0140 \\
\hline Income (\%) & & & & & 0.5452 \\
\hline Low & 832 & $25 \cdot 6$ & 1354 & $27 \cdot 0$ & \\
\hline Medium & 1884 & $51 \cdot 1$ & 2824 & $50 \cdot 1$ & \\
\hline High & 918 & $23 \cdot 3$ & 1409 & $23 \cdot 0$ & \\
\hline Educational level (\%) & & & & & $<0.0001$ \\
\hline$\leq 6$ years & 649 & 11.3 & 1694 & $24 \cdot 3$ & \\
\hline $6-12$ years & 1733 & $50 \cdot 8$ & 2368 & 45.4 & \\
\hline$\geq 12$ years & 1252 & $37 \cdot 8$ & 1525 & $30 \cdot 3$ & \\
\hline Smoking status (\%) & & & & & $<0.0001$ \\
\hline Never & 669 & $20 \cdot 4$ & 5013 & 87.5 & \\
\hline Former & 1561 & 34.5 & 314 & $6 \cdot 7$ & \\
\hline Current & 1404 & $45 \cdot 1$ & 260 & 5.7 & \\
\hline Alcohol intake (\%) & & & & & $<0.0001$ \\
\hline Never & 584 & $12 \cdot 7$ & 2002 & 31.0 & \\
\hline$<2$ times/week & 1692 & $52 \cdot 3$ & 3136 & $59 \cdot 7$ & \\
\hline$\geq 2$ times/week & 1358 & $35 \cdot 0$ & 449 & $9 \cdot 3$ & \\
\hline Physical activity (\%) & & & & & $<0.0001$ \\
\hline Regular & 1852 & 52.5 & 2512 & $46 \cdot 8$ & \\
\hline MetS (\%) & 1115 & $26 \cdot 4$ & 1377 & 20.5 & $<0.0001$ \\
\hline Hypertension (\%) & 1258 & $26 \cdot 2$ & 1507 & 21.2 & $<0.0001$ \\
\hline \multirow[t]{2}{*}{ Disease (\%) } & 576 & $10 \cdot 7$ & 681 & $10 \cdot 0$ & 0.4099 \\
\hline & Mean & SE & Mean & SE & $P \dagger$ \\
\hline Energy intake (g) & $2343 \cdot 6$ & $16 \cdot 2$ & 1705.5 & 11.2 & $<0.0001$ \\
\hline $\mathrm{Na}$ intake $(\mathrm{g})$ & $5953 \cdot 8$ & $70 \cdot 8$ & 4214.0 & $50 \cdot 3$ & $<0.0001$ \\
\hline DDS & $3 \cdot 8$ & 0.01 & 3.9 & 0.01 & $<0.0001$ \\
\hline BMI $\left(\mathrm{kg} / \mathrm{m}^{2}\right)$ & $24 \cdot 0$ & $0 \cdot 1$ & $23 \cdot 2$ & 0.1 & $<0.0001$ \\
\hline WC $(\mathrm{cm})$ & 84.0 & 0.2 & $77 \cdot 7$ & 0.2 & $<0.0001$ \\
\hline $\mathrm{SBP}(\mathrm{mmHg})$ & 118.9 & 0.4 & 114.5 & 0.4 & $<0.0001$ \\
\hline $\mathrm{DBP}(\mathrm{mmHg})$ & 77.5 & 0.3 & 72.5 & 0.2 & $<0.0001$ \\
\hline FPG (mmol/l) & $5 \cdot 5$ & 0.03 & $5 \cdot 2$ & 0.02 & $<0.0001$ \\
\hline TAG $(\mathrm{mmol} / \mathrm{l})$ & $1 \cdot 7$ & 0.03 & $1 \cdot 2$ & 0.02 & $<0.0001$ \\
\hline HDL-cholesterol (mmol/l) & 1.2 & 0.01 & $1 \cdot 3$ & 0.01 & $<0.0001$ \\
\hline
\end{tabular}

Disease, cancer, CVD and diabetes; MetS, metabolic syndrome; DDS, dietary diversity score; WC, waist circumference; SBP, systolic blood pressure; DBP, diastolic blood pressure;

FPG, fasting plasma glucose.

* $P$ value was determined by $x^{2}$ test.

$\dagger P$ value was determined by $t$ test. 
Table 2. Characteristics of the study subjects according to frequency of fried food consumption (Numbers and percentages; mean values with their standard errors)

\begin{tabular}{|c|c|c|c|c|c|c|c|c|c|}
\hline & \multicolumn{2}{|c|}{ Rarely* ( $n$ 3973) } & \multicolumn{2}{|c|}{$\geq 1 /$ month $(n$ 3802) } & \multicolumn{2}{|c|}{ 1/week ( $n$ 942) } & \multicolumn{2}{|c|}{$\geq 2 /$ week ( $n$ 504) } & \multirow[b]{2}{*}{$P \dagger$} \\
\hline & $n$ & $\%$ & $n$ & $\%$ & $n$ & $\%$ & $n$ & $\%$ & \\
\hline Age (years) & $54 \cdot 5^{\mathrm{a}}$ & 0.4 & $41.9^{\mathrm{b}}$ & 0.4 & $36 \cdot 3^{c}$ & 0.5 & $32 \cdot 0^{\mathrm{d}}$ & 0.5 & $<0.0001$ \\
\hline \multicolumn{9}{|l|}{ Sex $(\%)$} & $<0.0001$ \\
\hline Men & 1428 & 41.5 & 1519 & $48 \cdot 5$ & 446 & $57 \cdot 9$ & 241 & $59 \cdot 8$ & \\
\hline Women & 2545 & 58.5 & 2283 & 51.5 & 496 & $42 \cdot 1$ & 263 & $40 \cdot 2$ & \\
\hline \multicolumn{9}{|l|}{ Income (\%) } & 0.0260 \\
\hline Low & 1101 & 29.7 & 808 & 24.9 & 175 & $24 \cdot 4$ & 102 & $22 \cdot 8$ & \\
\hline Medium & 1965 & 48.9 & 2004 & $51 \cdot 6$ & 474 & $50 \cdot 7$ & 265 & $52 \cdot 2$ & \\
\hline High & 907 & 21.4 & 990 & $23 \cdot 6$ & 293 & 24.9 & 137 & $25 \cdot 0$ & \\
\hline \multicolumn{9}{|l|}{ Educational level (\%) } & $<0.0001$ \\
\hline$\leq 6$ years & 1701 & 35.4 & 569 & 11.3 & 64 & $5 \cdot 3$ & 9 & 0.9 & \\
\hline $6-12$ years & 1623 & $45 \cdot 1$ & 1789 & $48 \cdot 7$ & 430 & $48 \cdot 3$ & 259 & $56 \cdot 6$ & \\
\hline$\geq 12$ years & 649 & 19.5 & 1444 & $40 \cdot 1$ & 448 & $46 \cdot 4$ & 236 & $42 \cdot 5$ & \\
\hline \multicolumn{9}{|l|}{ Smoking status (\%) } & $<0.0001$ \\
\hline Never & 2541 & $58 \cdot 6$ & 2350 & 55.9 & 516 & $48 \cdot 0$ & 275 & $48 \cdot 0$ & \\
\hline Former & 845 & 21.8 & 755 & $19 \cdot 1$ & 186 & 18.9 & 89 & $20 \cdot 2$ & \\
\hline Current & 587 & $19 \cdot 6$ & 697 & $25 \cdot 0$ & 240 & 33.1 & 140 & 31.9 & \\
\hline \multicolumn{9}{|l|}{ Alcohol intake (\%) } & $<0.0001$ \\
\hline Never & 1471 & $31 \cdot 0$ & 870 & $19 \cdot 8$ & 166 & $13 \cdot 7$ & 79 & $10 \cdot 7$ & \\
\hline$<2$ times/week & 1775 & $47 \cdot 7$ & 2217 & $60 \cdot 0$ & 554 & 63.3 & 282 & 59.5 & \\
\hline$\geq 2$ times/week & 727 & $21 \cdot 3$ & 715 & $20 \cdot 2$ & 222 & $22 \cdot 9$ & 143 & 29.8 & \\
\hline \multicolumn{9}{|l|}{ Physical activity (\%) } & 0.0193 \\
\hline Regular & 1872 & 48.5 & 1761 & $48 \cdot 3$ & 465 & $52 \cdot 9$ & 266 & $55 \cdot 7$ & \\
\hline \multirow[t]{2}{*}{ Disease (\%) } & 773 & $16 \cdot 1$ & 411 & 8.8 & 57 & $4 \cdot 7$ & 16 & 3.0 & $<0.0001$ \\
\hline & Mean & SE & Mean & SE & Mean & SE & Mean & $\mathrm{SE}$ & $P \ddagger$ \\
\hline Energy intake (g) & $1850 \cdot 8^{a}$ & $16 \cdot 6$ & $2033.5^{\mathrm{b}}$ & $16 \cdot 0$ & $2251 \cdot 6^{c}$ & $33 \cdot 0$ & $2279 \cdot 1^{c}$ & $46 \cdot 7$ & $<0.0001$ \\
\hline Na intake $(g)$ & $4743 \cdot 1^{a}$ & $75 \cdot 0$ & $5105 \cdot 0^{\mathrm{b}}$ & $67 \cdot 2$ & $5521 \cdot 5^{c}$ & 132.5 & $5348 \cdot 6^{\mathrm{bc}}$ & $159 \cdot 4$ & $<0.0001$ \\
\hline DDS & $3.8^{a}$ & 0.02 & $3.9^{\mathrm{b}}$ & 0.01 & $3.9^{\mathrm{b}, \mathrm{c}}$ & 0.03 & $3 \cdot 8^{\mathrm{b}, \mathrm{d}}$ & 0.04 & $<0.0001$ \\
\hline $\mathrm{BMI}\left(\mathrm{kg} / \mathrm{m}^{2}\right)$ & $23 \cdot 7^{\mathrm{a}}$ & 0.1 & $23.5^{\mathrm{b}}$ & 0.1 & $23 \cdot 5^{a, b}$ & 0.1 & $23 \cdot 7^{\mathrm{a}, \mathrm{b}}$ & 0.2 & 0.1133 \\
\hline WC $(\mathrm{cm})$ & $81 \cdot 8^{\mathrm{a}}$ & 0.2 & $80 \cdot 1^{\mathrm{b}}$ & 0.3 & $80 \cdot 3^{\mathrm{b}}$ & 0.4 & $80 \cdot 1^{\mathrm{b}}$ & 0.7 & $<0.0001$ \\
\hline $\mathrm{SBP}(\mathrm{mmHg})$ & $121 \cdot 7^{a}$ & 0.5 & $114.5^{\mathrm{b}}$ & 0.4 & $113 \cdot 1^{\mathrm{c}}$ & 0.6 & $111 \cdot 8^{\mathrm{c}}$ & 0.6 & $<0.0001$ \\
\hline DBP $(\mathrm{mmHg})$ & $75 \cdot 7^{\mathrm{a}}$ & 0.3 & $74.5^{\mathrm{b}}$ & 0.3 & $74 \cdot 3^{\mathrm{b}}$ & 0.5 & $74 \cdot 3^{\mathrm{b}}$ & 0.5 & 0.0014 \\
\hline $\mathrm{FPG}(\mathrm{mmol} / \mathrm{l})$ & $5 \cdot 5^{\mathrm{a}}$ & 0.03 & $5 \cdot 3^{\mathrm{b}}$ & 0.02 & $5 \cdot 2^{c}$ & 0.04 & $5 \cdot 2^{b, c}$ & 0.09 & $<0.0001$ \\
\hline TAG $(\mathrm{mmol} / \mathrm{l})$ & $1.5^{\mathrm{a}}$ & 0.02 & $1.4^{\mathrm{b}}$ & 0.03 & $1.5^{\mathrm{a}, \mathrm{b}}$ & 0.05 & $1.5^{\mathrm{a}, \mathrm{b}}$ & 0.09 & 0.0060 \\
\hline HDL-cholesterol (mmol/l) & $1 \cdot 2^{\mathrm{a}}$ & 0.01 & $1.3^{\mathrm{b}}$ & 0.01 & $1.3^{\mathrm{b}}$ & 0.01 & $1.3^{\mathrm{b}}$ & 0.02 & 0.0004 \\
\hline
\end{tabular}

higher BMI, WC, SBP, DBP, FPG and TAG compared with women, whereas HDL-cholesterol was significantly lower in men than in women $(P<0.0001)$. The prevalence of the MetS and hypertension were significantly higher in men than in women $(P<0 \cdot 0001)$.

\section{Characteristics of subjects according to frequency of fried food consumption}

Characteristics of subjects according to frequency of fried food consumption are shown in Table 2 . Subjects in the highest category of fried food consumption $(\geq 2 /$ week) were younger and more likely to be men compared with those in the lowest category (rarely). Subjects in the highest category of fried food consumption were more likely to have a higher income, higher educational level, be non-smokers, alcohol drinkers, exercise regularly and to have higher $\mathrm{Na}$ intake. In terms of healthrelated variables, subjects in the highest category of fried food consumption were more likely to have lower SBP, DBP and FPG and higher HDL-cholesterol level.

\section{Associations of frequency of fried food consumption with metabolic risk factors and hypertension}

Adjusted OR and $95 \%$ CI for components of the MetS according to frequency of fried food consumption are showed in Table 3. The adjusted OR of elevated BP increased by 1.6-fold in men $\left(P_{\text {trend }}=0.0447\right)$ and by $2 \cdot 2$-fold in women $\left(P_{\text {trend }}=0.0403\right)$ who reported eating fried food more than twice a week compared with those who reported rarely consuming fried food after adjusting for potential risk factors of age, BMI, income level, education level, smoking status, alcohol intake, physical activity, total energy intake, Na intake, DDS and the presence of chronic disease. However, fried food consumption was not associated with other metabolic risk factors. Adjusted OR and $95 \%$ CI for hypertension according to frequency of fried food 
Table 3. The metabolic risk factors and hypertension according to fried food consumption (Odds ratios and $95 \%$ confidence intervals)

\begin{tabular}{|c|c|c|c|c|c|c|c|c|c|}
\hline & \multirow{2}{*}{$\frac{\text { Rarely* }}{\text { Ref. }}$} & \multicolumn{2}{|c|}{$\geq 1 /$ month } & \multicolumn{2}{|c|}{$1 /$ week } & \multicolumn{2}{|c|}{$\geq 2 /$ week } & \multirow[b]{2}{*}{$P$} & \multirow[b]{2}{*}{$P \dagger$} \\
\hline & & 0 & $95 \% \mathrm{Cl}$ & OR & $95 \% \mathrm{Cl}$ & OR & $95 \% \mathrm{Cl}$ & & \\
\hline \multicolumn{10}{|l|}{ Totalł } \\
\hline Abdominal obesity§ & 1.0 & 0.91 & $0.77,1.07$ & 0.74 & $0.58,0.96$ & 0.65 & $0.47,0.88$ & 0.7307 & 0.0157 \\
\hline High fasting glucose & 1.0 & 1.05 & $0.91,1.22$ & $1 \cdot 11$ & $0.86,1.44$ & 0.92 & $0.64,1.33$ & 0.6742 & 0.1288 \\
\hline Hypertriacylglycerolaemia & 1.0 & 1.08 & $0.94,1.25$ & 0.89 & $0.72,1.10$ & 1.22 & $0.92,1.62$ & 0.1611 & 0.7533 \\
\hline Low HDL-cholesterol & 1.0 & 1.06 & $0.92,1.22$ & 0.96 & $0.77,1.20$ & 0.85 & $0.66,1.10$ & 0.2937 & 0.9311 \\
\hline Elevated BP\| & 1.0 & 1.23 & $1.06,144$ & 1.16 & $0.91,1.49$ & 1.56 & $1 \cdot 14,2 \cdot 15$ & 0.0126 & $<0.0001$ \\
\hline MetS & 1.0 & $1 \cdot 16$ & $0.99,1.36$ & 0.93 & $0.71,1.23$ & 0.98 & $0.65,1.49$ & 0.2368 & 0.2048 \\
\hline \multicolumn{10}{|l|}{ Menף } \\
\hline Abdominal obesity** & 1.0 & 0.92 & $0 \cdot 70,1 \cdot 21$ & 0.83 & $0.56,1.24$ & 0.65 & $0.42,1.02$ & 0.2733 & \\
\hline High fasting glucose & 1.0 & 1.15 & $0.92,1.44$ & 0.97 & $0.70,1.36$ & 1.07 & $0.64,1.80$ & 0.5589 & \\
\hline Hypertriacylglycerolaemia & 1.0 & 1.18 & $0.95,1.46$ & 1.01 & $0.74,1.37$ & 1.40 & $0.96,2.05$ & 0.2056 & \\
\hline Low HDL-cholesterol & 1.0 & 1.06 & $0.85,1.33$ & 0.394 & $0.67,1.31$ & 0.78 & $0.51,1.18$ & 0.4384 & \\
\hline Elevated BP\| & 1.0 & 1.27 & $1.01,1.59$ & $1 \cdot 12$ & $0.81,156$ & 1.62 & $1 \cdot 11,2.37$ & 0.0447 & \\
\hline MetS & 1.0 & 1.23 & $0.97,1.56$ & 0.94 & $0.63,1.38$ & 1.06 & $0.63,1.79$ & 0.2430 & \\
\hline \multicolumn{10}{|l|}{ Womeng } \\
\hline Abdominal obesity** & 1.0 & 0.84 & $0.70,1.02$ & 0.61 & $0.44,0.84$ & 0.75 & $0.44,1.27$ & 0.0214 & \\
\hline High fasting glucose & 1.0 & 0.95 & $0.76,1.19$ & 1.49 & $0.98,2.27$ & 0.67 & $0.42,1.09$ & 0.0551 & \\
\hline Hypertriacylglycerolaemia & 1.0 & 0.92 & $0.74,1.16$ & $0 \cdot 70$ & $0.48,1.03$ & 1.07 & $0.60,1.89$ & 0.3131 & \\
\hline Low HDL-cholesterol & 1.0 & 1.06 & $0.89,1.26$ & 0.98 & $0.75,1.28$ & 0.97 & $0.71,1.33$ & 0.8219 & \\
\hline Elevated BP\| & 1.0 & 1.07 & $0.86,133$ & 1.42 & $0.94,2.15$ & $2 \cdot 20$ & $1.21,4.00$ & 0.0403 & \\
\hline MetS & 1.0 & 1.02 & $0.79,1.32$ & 0.99 & $0.62,1.60$ & 0.97 & $0.49,1.90$ & 0.9983 & \\
\hline
\end{tabular}

Ref., referent values; BP, blood pressure; MetS, metabolic syndrome

* Rarely: rarely or 6-1/year, $\geq 1$ /month: $1-3 /$ month, $1 /$ week: $1 /$ week, $\geq 2 /$ week: $2-6 /$ week or $1-3 /$ d.

$\dagger P$ for interaction between sex.

$\ddagger$ Adjusted for age, sex, BMl, income level, education level, smoking status, alcohol consumption, physical activity, energy intake, dietary diversity score and disease.

$\S$ Adjusted for modelł excluding BMI.

IAdjusted for model $\$$, I plus Na intake.

TI Adjusted for age, BMI, income level, education level, smoking status, alcohol consumption, physical activity, energy intake, dietary diversity score and disease.

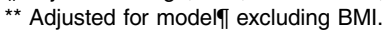

Table 4. Blood pressure and hypertension according to frequency of fried food consumption

(Odds ratios and $95 \%$ confidence intervals)

\begin{tabular}{|c|c|c|c|c|c|c|c|c|c|}
\hline & \multirow{2}{*}{$\frac{\text { Rarely }}{\text { Ref. }}$} & \multicolumn{2}{|c|}{$\geq 1 /$ month } & \multicolumn{2}{|c|}{$1 /$ week } & \multicolumn{2}{|c|}{$\geq 2 /$ week } & \multirow[b]{2}{*}{$P$} & \multirow[b]{2}{*}{$P^{*}$} \\
\hline & & OR & $95 \% \mathrm{Cl}$ & OR & $95 \% \mathrm{Cl}$ & OR & $95 \% \mathrm{Cl}$ & & \\
\hline \multicolumn{10}{|l|}{ Hypertension } \\
\hline Unadjusted & 1.0 & 2.55 & $2 \cdot 21,2 \cdot 94$ & 3.53 & $2 \cdot 75,4.55$ & 5.67 & $3.99,8.06$ & $<0.0001$ & $<0.0001$ \\
\hline Model 1† & 1.0 & $1 \cdot 14$ & $0.97,1.34$ & 1.03 & $0.79,1.35$ & $1 \cdot 18$ & $0.78,1.79$ & 0.4327 & $<0.0001$ \\
\hline Model $2 \ddagger$ & 1.0 & $1 \cdot 18$ & $0.99,1.39$ & $1 \cdot 11$ & $0.84,1.46$ & 1.41 & $0.93,2.14$ & 0.1799 & $<0.0001$ \\
\hline \multicolumn{10}{|l|}{ Men } \\
\hline Unadjusted & 1.0 & 2.00 & $1.61,2.49$ & 2.55 & $1.84,3.52$ & 3.78 & $2.48,5.74$ & $<0.0001$ & \\
\hline Model 1§ & 1.0 & 1.09 & $0.86,1.38$ & 0.94 & $0.67,1.30$ & $1 \cdot 16$ & $0.73,1.83$ & 0.6788 & \\
\hline Model $2 \|$ & 1.0 & $1 \cdot 13$ & $0.89,1.44$ & 0.99 & $0.70,1.39$ & 1.39 & $0.87,2.23$ & 0.4243 & \\
\hline \multicolumn{10}{|l|}{ Women } \\
\hline Unadjusted & 1.0 & 3.43 & $2.88,4.07$ & 7.54 & $5.06,11.25$ & $23 \cdot 17$ & $11 \cdot 87,45 \cdot 23$ & $<0.0001$ & \\
\hline Model 1§ & 1.0 & $1 \cdot 10$ & $0.89,1.37$ & 1.67 & $1.06,2 \cdot 62$ & $2 \cdot 27$ & $1 \cdot 16,4.42$ & 0.0219 & \\
\hline Model $2 \|$ & 1.0 & $1 \cdot 14$ & $0.91,1.43$ & $1 \cdot 62$ & $1.01,2.58$ & $2 \cdot 37$ & $1 \cdot 19,4 \cdot 72$ & 0.0272 & \\
\hline
\end{tabular}

Ref., referent values.

* $P$ for interaction between sex.

$\dagger$ Adjusted for age and sex.

$\ddagger$ Model $1+\mathrm{BMI}$, income level, educational level, smoking status, alcohol consumption, physical activity, energy intake, Na intake, dietary diversity score and disease.

$\S$ Adjusted for age.

|| Model 2 + BMl, income level, educational level, smoking status, alcohol consumption, physical activity, energy intake, Na intake, dietary diversity score and disease.

consumption are shown in Table 4. For women, the OR of hypertension showed a $2 \cdot 4$-fold increase in those reporting fried food consumption of more than twice a week compared with those who reported rarely eating fried food after adjusting for potential risk factors $\left(P_{\text {trend }}=0.0272\right)$ and potential confounders. No significant association was found between the frequency of fried food consumption and hypertension in men.

\section{Discussion}

This study found that fried food consumption was strongly associated with hypertension among Korean women. For men, the OR of elevated BP showed a 1.6-fold increase after adjusting for potential risk factors of age, BMI, income level, education level, smoking status, alcohol intake, physical activity, total 
energy intake, $\mathrm{Na}$ intake, DDS and the presence of chronic disease for those reporting a fried food consumption more than twice a week compared with those who reported rarely eating fried foods. Women also showed a $2 \cdot 2$-fold increase in the OR of elevated BP for those reporting fried food consumption more than twice a week compared with those who reported rarely eating fried food after adjusting for potential risk factors. In addition, only women showed a $2 \cdot 4$-fold increase in the OR of hypertension among those who reported eating fried food more than twice a week compared with those who reported rarely eating fried food after adjusting for potential risk factors.

Our findings are similar to results from a previous study by Sayon et al. ${ }^{(10)}$ involving 8289 Spanish men and women aged 20-90 years, which reported a positive association between fried food consumption and the prevalence of elevated BP. Sayon et al. ${ }^{(10)}$ found that subjects in the upper category of fried food consumption had a 1.16-fold higher risk of elevated BP compared with those in the lower category of fried food consumption. Among 5037 US adults aged 65 years and older, participants consuming fried fish more than once a week tended to have an increased risk of elevated BP compared with those consuming fried fish less than once a month, suggesting that fried food intake is related to higher mean $\mathrm{BP}^{(24)}$. A Mediterranean cohort study on 13679 Spanish university graduates found that those who consumed fried foods more than four times a week had a 1.21-fold greater risk of hypertension compared with those eating fried foods less than twice a week $^{(13)}$

Several reports have demonstrated the mechanisms behind the relationship between fried food consumption and elevated $\mathrm{BP}$ or hypertension. The process of frying involves various physicochemical changes and reactions including oxidation, pyrolysis, polymerisation, hydrolysis and isomerisation. A multitude of products formed by frying, such as polar compounds resulting from oils and fats heated at high temperatures, may influence $\mathrm{BP}^{(25,26)}$. In particular, a considerable amount of trans-fatty acids (TFA) is produced during the process of frying ${ }^{(26,27)}$. TFA elevate LDL-cholesterol level and reduce HDL-cholesterol level. Increased LDL-cholesterol increases the expression of angiotensin II (Ang II) type 1 receptors, which explains the increased sensitivity to Ang II in vascular smooth muscle cells ${ }^{(28)}$. Ang II leads to contraction of blood vessels and increases BP by inducing excretion of water and salt from the kidney ${ }^{(29)}$. In this way, TFA contribute to elevated BP. In Iranian women aged 40-60 years, a higher intake of TFA was associated with hypertension in a cross-sectional study ${ }^{(30)}$. A randomised controlled study reported that higher intakes of TFA and SFA were associated with increased risk of hypertension in middle-aged and older women ${ }^{(31)}$.

In this study, there were no associations between fried food and other metabolic risk factors - such as abdominal obesity, FPG and lipid level - which are not consistent with some other studies $^{(32,33)}$. This may be due to differences in the frequency of fried food consumption of subjects, the type of food group and the cooking method among studies. The subjects from our study may consume less fried food compared with other population $^{(32,33)}$. Our study compared the Korean adults consuming fried food more than twice a week with those consuming it rarely, whereas the previous study showed that US adults consuming fried food more than four times a week or seven times a week had higher risk of type 2 diabetes in comparison with those consuming it rarely ${ }^{(33)}$. More frequent consumption of fried food may be associated with blood glucose. The type of food group might contribute to the inconsistent results. According to the data from 24-h recall, fried meat ( $45 \%$ ), vegetables (31\%), fish (12\%) and seaweed ( $8 \%$ ) were the four groups of fried food most frequently consumed by Korean adults, whereas fried meat, fish, potatoes and eggs were the four groups of fried food most frequently consumed by Spanish adults, with $75 \%$ of subjects consuming each of those groups of food reporting a positive association between fried meat, fish and eggs intake and general obesity/central obesity $^{(32)}$. Therefore, high consumption of plant food groups with less energy such as vegetable and seaweed in Korean population might have attenuated the relationship between fried food and abdominal obesity. Furthermore, cooking method - deep-frying or pan-frying - could have affected the relationship between fried food intake and metabolic risk factors. The frying method have different effects on the absorption of fat depending on the food, the oil used and the frying conditions ${ }^{(34)}$. However, this study could not distinguish between the two frying methods.

Interestingly, the present study showed an association between fried food consumption and hypertension in women only. It has been suggested that there are sex differences in the relationship between hypertension and its risk factors. Risk factors for hypertension such as renin-angiotensin system, sex hormones and increased immune inflammatory factors may have a greater impact on women than on $\operatorname{men}^{(35)}$. The responses to a stimulation of renin-angiotensin system were different in men and in women ${ }^{(36)}$. Likewise, women may be more sensitive compared with men to the relationship between diet and hypertension. The effect of diet or nutrients on BP may be greater in women than in men. For example, the reduction in $\mathrm{BP}$ with a diet of reduced $\mathrm{Na}$ and $\mathrm{K}$ supplementation was greater in women than in men ${ }^{(35)}$. Abramson et al. ${ }^{(37)}$ reported that a modified-lifestyle approach involving a healthy diet was effective in mitigating risk factors for hypertension in women. Additionally, hypertension may be more difficult to manage in women than in men. A recent study on patients with hypertension documented that women were less likely to have BP control compared with men, although there were no differences in the use of BP medication or initiation of a new therapy for patients with uncontrolled hypertension, suggesting that the sex-based differences in BP treatment are necessary ${ }^{(38)}$. Besides, there are many different pathophysiological and social reasons why the results may have been only significant in women - which researchers are unable to specifically determine in the present study - but, that regardless, the results suggest that reducing fried food intake may be one method to decrease risk for hypertension in women.

The present study has several limitations. First, the results are not able to verify a causal relationship between fried food consumption and hypertension, because the study was crosssectional in design. There is a possibility that subjects with hypertension might change their lifestyle such as diet and 
physical activity. Second, we did not investigate in detail the type of fat or oil used for frying. In spite of these limitations, to the best of our knowledge, this is the first study to reveal associations among fried food consumption and hypertension and metabolic risk factors in a Korean population using data from a large nationally representative sample.

In conclusion, frequent consumption of fried food more than twice a week was associated with hypertension after adjusting for potential confounders in Korean women. According to this result, we suggest reducing fried food consumption and changing cooking methods to broiling, steaming and boiling. Less frequent consumption of fried foods and modifying cooking methods may help decrease elevated $\mathrm{BP}$ and reduce the risk of hypertension. A study on the effect of fat or oil type on health outcome and its underlying mechanism requires a better understanding of the associations between fried food consumption and chronic diseases in further studies.

\section{Acknowledgements}

We thank all study participants and staffs who participated in KNHANES

This research was supported by the Basic Science Research Program of the National Research Foundation of Korea (NRF) founded by the Ministry of Education, Science and Technology (NRF2012R1A1A1012317).

The authors' contributions are as follows: Y. K. helped with the acquisition, analysis and interpretation of the data and wrote the paper. J. K. contributed to research design, analysis and interpretation of the data, writing of the paper and had primary responsibility for the final content. All authors read and approved the final manuscript.

None of the authors has any conflicts of interest.

\section{References}

1. Kearney PM, Whelton M, Reynolds K, et al. (2004) Worldwide prevalence of hypertension: a systematic review. I Hypertens 22, 11-19.

2. Kim HJ, Kim Y, Cho Y, et al. (2014) Trends in the prevalence of major cardiovascular disease risk factors among Korean adults: results from the Korea National Health and Nutrition Examination Survey, 1998-2012. Int J Cardiol 174, 64-72.

3. Srinath Reddy K \& Katan MB (2004) Diet, nutrition and the prevention of hypertension and cardiovascular diseases. Public Health Nutr 7, 167-186.

4. Houston MC (2013) The role of nutrition, nutraceuticals, vitamins, antioxidants, and minerals in the prevention and treatment of hypertension. Altern Ther Health Med 19, Suppl. 1, $32-49$.

5. Park JE, Jung H \& Lee JE (2014) Dietary pattern and hypertension in Korean adults. Public Health Nutr 17, 597-606.

6. Lichtenstein AH (2014) Dietary trans fatty acids and cardiovascular disease risk: past and present. Curr Atheroscler Rep 16, 433

7. Bordin K, Kunitake MT, Aracava KK, et al. (2013) Changes in food caused by deep fat frying - a review. Arch Latinoam Nutr 63, 5-13.

8. İnanç T \& Maskan M (2014) Effect of carvacrol on the oxidative stability of palm oil during frying. Grasas Aceites 65, e042.
9. Donfrancesco C, Lo Noce C, Brignoli O, et al. (2008) Italian network for obesity and cardiovascular disease surveillance: a pilot project. BMC Fam Pract 9, 53.

10. Sayon-Orea C, Martinez-Gonzalez MA, Gea A, et al. (2014) Consumption of fried foods and risk of metabolic syndrome: the SUN cohort study. Clin Nutr 33, 545-549.

11. Kelishadi R, Pour MH, Zadegan NS, et al. (2004) Dietary fat intake and lipid profiles of Iranian adolescents: Isfahan Healthy Heart Program - heart health promotion from childhood. Prev Med 39, 760-766.

12. Lutsey PL, Steffen LM \& Stevens J (2008) Dietary intake and the development of the metabolic syndrome: the Atherosclerosis Risk in Communities study. Circulation 117, 754-761.

13. Sayon-Orea C, Bes-Rastrollo M, Gea A, et al. (2014) Reported fried food consumption and the incidence of hypertension in a Mediterranean cohort: the SUN (Seguimiento Universidad de Navarra) project. Br J Nutr 112, 984-991.

14. Kim S, Moon S \& Popkin BM (2000) The nutrition transition in South Korea. The Am J Clin Nutr 71, 44-53.

15. Kim DW, Song S, Lee JE, et al. (2015) Reproducibility and validity of an FFQ developed for the Korea National Health and Nutrition Examination Survey (KNHANES). Public Health Nutr 18, 1369-1377.

16. Ministry of Health and Welfare (2005) National Health and Nutrition Examination Survey Report 2005. Seoul: Ministry of Health and Welfare.

17. Kim J \& Jo I (2010) Relationship between body mass index and alanine aminotransferase concentration in non-diabetic Korean adults. Eur J Clin Nutr 64, 169-175.

18. Nutrition TASo (1992) Recommendations for routine blood pressure measurement by indirect cuff sphygmomanometry. American Society of Hypertension. Am J Hypertens 5, 207-209.

19. Alberti KG, Eckel RH, Grundy SM, et al. (2009) Harmonizing the metabolic syndrome: a joint interim statement of the International Diabetes Federation Task Force on Epidemiology and Prevention; National Heart, Lung, and Blood Institute; American Heart Association; World Heart Federation; International Atherosclerosis Society; and International Association for the Study of Obesity. Circulation 120, 1640-1645.

20. Kim D \& Kim J (2015) Association of serum 25-hydroxyvitamin D and parathyroid hormone with hypertension in middle-aged and older Korean adults. Am J Hypertens (epublication ahead of print version 23 April 2015).

21. Kim J (2013) Dairy food consumption is inversely associated with the risk of the metabolic syndrome in Korean adults. J Hum Nutr Diet 26, Suppl. 1, 171-179.

22. Kant AK (1996) Indexes of overall diet quality: a review. J Am Diet Assoc 96, 785-791.

23. Hernan MA, Hernandez-Diaz S, Werler MM, et al. (2002) Causal knowledge as a prerequisite for confounding evaluation: an application to birth defects epidemiology. Am J Epidemiol 155, 176-184.

24. Mozaffarian D, Gottdiener JS \& Siscovick DS (2006) Intake of tuna or other broiled or baked fish versus fried fish and cardiac structure, function, and hemodynamics. Am J Cardiol 97, 216-222

25. Soriguer F, Rojo-Martinez G, Dobarganes MC, et al. (2003) Hypertension is related to the degradation of dietary frying oils. Am J Clin Nutr 78, 1092-1097.

26. Bhardwaj S, Passi SJ \& Misra A (2011) Overview of trans fatty acids: biochemistry and health effects. Diabetes Metab Syndr 5, 161-164.

27. Micha R \& Mozaffarian D (2008) Trans fatty acids: effects on cardiometabolic health and implications for policy. Prostaglandins Leukot Essent Fatty Acids 79, 147-152. 
28. van der Linde NA, Sijbrands EJ, Boomsma F, et al. (2006) Effect of low-density lipoprotein cholesterol on angiotensin II sensitivity: a randomized trial with fluvastatin. Hypertension $\mathbf{4 7}$, $1125-1130$.

29. Alpert MA, Govindarajan G, Del Rosario ML, et al. (2009) The role of the renin-angiotensin system in the pathophysiology, prevention, and treatment of renal impairment in patients with the cardiometabolic syndrome or its components. J Cardiometab Syndr 4, 57-62.

30. Esmaillzadeh A \& Azadbakht L (2011) Different kinds of vegetable oils in relation to individual cardiovascular risk factors among Iranian women. Br J Nutr 105, 919-927.

31. Wang L, Manson JE, Forman JP, et al. (2010) Dietary fatty acids and the risk of hypertension in middle-aged and older women. Hypertension 56, 598-604.

32. Guallar-Castillon P, Rodriguez-Artalejo F, Fornes NS, et al. (2007) Intake of fried foods is associated with obesity in the cohort of Spanish adults from the European Prospective Investigation into Cancer and Nutrition. Am J Clin Nutr 86, 198-205.
33. Cahill LE, Pan A, Chiuve SE, et al. (2014) Fried-food consumption and risk of type 2 diabetes and coronary artery disease: a prospective study in 2 cohorts of US women and men. Am J Clin Nutr 100, 667-675.

34. Andrikopoulos NK, Dedoussis GV, Falirea A, et al. (2002) Deterioration of natural antioxidant species of vegetable edible oils during the domestic deep-frying and pan-frying of potatoes. Int J Food Sci Nutr 53, 351-363.

35. Balan H \& Popescu L (2014) 'Gender specific medicine': a focus on gender-differences in hypertension. Rom J Intern Med 52, 129-141.

36. Pechere-Bertschi A \& Burnier M (2004) Female sex hormones, salt, and blood pressure regulation. Am J Hypertens 17, 994-1001.

37. Abramson BL \& Melvin RG (2014) Cardiovascular risk in women: focus on hypertension. Can J Cardiol 30, 553-559.

38. Keyhani S, Scobie JV, Hebert PL, et al. (2008) Gender disparities in blood pressure control and cardiovascular care in a national sample of ambulatory care visits. Hypertension 51, 1149-1155. 